\title{
Réplique à l'article de Michèle Dagenais et Christian Laville
}

\section{Robert Comeau et Josiane Lavallée}

Volume 61, numéro 2, automne 2007

URI : https://id.erudit.org/iderudit/018062ar

DOI : https://doi.org/10.7202/018062ar

Aller au sommaire du numéro

Éditeur(s)

Institut d'histoire de l'Amérique française

ISSN

0035-2357 (imprimé)

1492-1383 (numérique)

Découvrir la revue

Citer cet article

Comeau, R. \& Lavallée, J. (2007). Réplique à l'article de Michèle Dagenais et Christian Laville. Revue d'histoire de l'Amérique française, 61(2), 253-259.

https://doi.org/10.7202/018062ar d'utilisation que vous pouvez consulter en ligne.

https://apropos.erudit.org/fr/usagers/politique-dutilisation/ 


\title{
Débat
}

\section{Réplique à l'article de Michèle Dagenais et Christian Laville}

\author{
ROBERT COMEAU \\ Département d'histoire \\ Université du Québec à Montréal \\ Membre du CA de la Société des professeurs d'histoire du Québec \\ JOSIANE LAVALLÉE \\ Historienne et membre du CA de la Société des professeurs \\ d'histoire du Québec
}

\begin{abstract}
À la suite de la lecture de l'article de Michèle Dagenais et Christian LLaville publié dans les pages de la Revue d'histoire de l'Amérique française du printemps dernier au sujet de la polémique entourant la réforme du programme en histoire et éducation à la citoyenneté en secondaire 3 et 4 , nous aimerions rectifier certains propos tenus à notre égard, préciser les raisons de nos désaccords avec le contenu du projet de programme et clarifier notre position dans ce débat.
\end{abstract}

\section{CITATIONS TRONQUÉES ET INTERPRÉTATIONS ABUSIVES}

Dans leur texte, Dagenais et Laville nous dépeignent comme étant «les plus farouches défenseurs du récit traditionnel de l'histoire du Québec», en déformant ce que nous avions écrit. Alors que nous disions: «on a pu constater à quel point les Québécois tenaient à conserver un enseignement de l'histoire fondé sur les événements qui ont imprimé un souvenir indélébile dans leur mémoire nationale et dans leur imaginaire collectif ${ }^{\text {» }}$.

1. Voir à ce sujet, notre introduction au livre L'historien Maurice Séguin. Théoricien de l'indépendance et penseur de la modernité québécoise (Sillery, Septentrion, 2006), 14. 
Dagenais et Laville, en tronquant notre citation, laissent croire au lecteur que c'est nous qui : «reconnaiss[ons] vouloir conserver un enseignement de l'histoire fondé sur les événements qui ont imprimé un souvenir indélébile dans [la] mémoire nationale et dans [l']imaginaire collectif » et non les Québécois comme nous l'entendions. À la suite de cette citation déformée, les auteurs concluent que «la polémique aura surtout été alimentée par des craintes de nationalistes et, qui plus est, de nationalistes attachés à une vision ethnique de la nation. Parce que ne pouvant concevoir un enseignement de l'histoire du Québec non nationaliste, toute autre proposition ne pouvait apparaître que suspecte - comprendre fédéraliste - à leurs yeux». Bien que nous ayons critiqué la conception de la nation civique de Gérard Bouchard en soulignant que l'ouverture aux autres ne devait pas entraîner l'effacement de la mémoire nationale des Québécois d'origine canadienne-française, cela ne fait pas de nous des nationalistes préconisant une vision ethnique de la nation québécoise.

Selon les deux auteurs, nous souhaiterions revenir "à une trame événementielle traditionnelle » de l'histoire du Québec dans le but de prôner une « histoire nationaliste» rattachée à des faits et des dates correspondant à « des conflits». En quoi vouloir enseigner la trame historique impliquant les événements de 1760, 1837, 1840 et 1867 serait-elle en soit condamnable et mériterait-elle l'étiquette de trame traditionnelle? Pourquoi ces dates fondamentales de l'histoire politique et nationale du Québec, qui ont aussi des dimensions socio-économiques et qui sont aussi des dates importantes au Canada, devraient-elles être évacuées?

Par ailleurs, Dagenais et Laville nous accusent de vouloir faire de la propagande en tirant des leçons du passé. Plus précisément, ils écrivent: «toutes ces dates et tous ces faits correspondent à des conflits et des tensions dont on veut tirer des enseignements. Il s'agit, expliquent deux adversaires du programme, d'autant de "leçons du passé" ". À nouveau Dagenais et Laville citent hors contexte ce que nous avons écrit dans notre introduction à notre ouvrage sur Maurice Séguin: «le Parti Québécois rend publique une déclaration de souveraineté fondée sur les leçons du passé... qui reprend l'interprétation néonationaliste sur les conséquences de la Conquête et ses suites». L'expression «leçons du passé» n'est pas de nous, mais du journaliste Michel Venne qui publiait, le jeudi 7 septembre 1995, à la une du journal Le Devoir «Une déclaration de souveraineté fondée sur les leçons du passé».

Aux yeux de Dagenais et Laville, nous serions donc pour le retour d'un "récit nationaliste composé de luttes et de revers à transmettre d'une génération à l'autre» dans le but de leur présenter «l'histoire comme le 
récit d'un itinéraire historique inachevé, on les invite[rait] à le conclure». Ainsi, Dagenais et Lavillle nous accusent de vouloir orienter l'enseignement de l'histoire du Québec pour promouvoir l'indépendance du Québec auprès des jeunes Québécois. À en croire nos deux auteurs, il faudrait éviter de parler de l'oppression nationale du Québec par crainte de manquer d'objectivité, comme s'il était impossible d'aborder ce phénomène sans nécessairement tomber dans les ornières du nationalisme ou pire de contribuer à l'orientation de l'avenir du Québec. Quelle honte y a-t-il à vouloir transmettre les luttes de générations successives de Québécois aux élèves de toutes origines? Pourquoi devrions-nous évacuer toute trace de luttes nationales et politiques du passé québécois? Une nation peut-elle se construire sur l'oubli de son passé?

\section{DEUX CONCEPTIONS DE L'HISTOIRE}

À notre avis, nous pensons que le litige dans ce débat se situe ailleurs qu'entre partisans de la nation civique et de la nation ethnique, comme les auteurs de l'article le prétendent. Il s'agit plutôt, selon nous, d'une opposition de deux conceptions de l'histoire : une conception de l'histoire du Québec qui occulte de l'enseignement les événements politiques qui ont façonné le parcours identitaire des Québécois et une approche qui tient compte des dimensions politique et nationale. Car nous sommes bien conscients, comme l'explique Marc Ferro, que «l'histoire a toujours servi à façonner l'identité d'une nation». En voulant écarter de l'enseignement de l'histoire les événements reliés aux rapports du Québec avec l'Empire britannique puis avec la majorité anglophone, peut-on croire que les auteurs n'ont pas souhaité promouvoir l'identité de l'autre nation qui est majoritaire au Canada?

Ce que nous déplorons dans le projet de programme, ce n'est pas l'absence d'une histoire nationaliste mais l'absence d'une histoire comportant des dimensions politiques et nationales. D’où notre profond désaccord avec ce contenu de programme qui faisait fi d'événements incontournables de l'histoire du Québec et du Canada. Il est intéressant de rappeler ici que même l'historien de métier Gérard Bouchard, présenté comme le modèle de promoteur du nationalisme civique, a signé la pétition qu'a fait circuler l'étudiante à la maîtrise Julie Guyot en mai 2006 qui dénonçait le contenu de ce projet de programme. Le camp des opposants ne regroupait pas que des nationalistes frileux et ethnicistes.

En dénonçant le contenu du projet de programme, nous avons voulu démontrer que des tenants de l'histoire révisionniste et les postnationa- 
listes (comme Jocelyn Létourneau qui nie même l'existence de la nation québécoise), ne voulaient plus que s'enseignent dans les écoles québécoises des événements politiques, qui intéressent aussi le Canada dans son ensemble, comme la Conquête, les Rébellions de 1837-1838, l'Acte d'Union de 1840 et la Fédération de 1867, car cela serait porteur de divisions. Ce faisant les chantres d'une vision «rassembleuse » et "plurielle» de l'histoire du Québec, ces prétendus avant-gardistes révisionnistes et postnationalistes qui nous invitent «à tenir compte de la contribution des Amérindiens et des groupes issus de l'immigration à l'histoire commune » voudraient occulter de la mémoire nationale des Québécois leur parcours distinctif en Amérique. Comme si le fait d'enseigner les causes et les conséquences de 1760,1837, 1840 et 1867, nous empêchait d'inclure dans cet enseignement l'apport des peuples autochtones et des différentes communautés ethnoculturelles à l'histoire du Québec.

\section{POUR UNE HISTOIRE DIVERSIFIÉE, RESPECTUEUSE DU PASSÉ QUÉBÉCOIS}

En 1996, lors du dépôt du rapport Lacoursière sur l'enseignement de l'histoire, nous nous réjouissions de voir que l'histoire serait enseignée chaque année du secondaire; ce qui permettrait d'accorder davantage de temps à l'enseignement de l'histoire du Québec au niveau politique, social, économique et culturel et de tenir compte des nouvelles recherches venues enrichir l'historiographie québécoise depuis trente ans. On n'a qu'à penser aux nombreuses recherches en histoire des femmes, des communautés ethnoculturelles, des peuples autochtones, du monde ouvrier et syndical, sans oublier l'histoire des idéologies, des mentalités, de la culture et plus récemment de l'histoire militaire et des relations internationales.

Comment Dagenais et Laville peuvent-ils prétendre que nous voulons évacuer toutes ces facettes de l'histoire du Québec? Nous pensons qu'il est tout à fait possible de diversifier le contenu historique des programmes sans avoir à renier des pans entiers de l'histoire du Québec. Ils prétendent sans aucune démonstration que nous serions opposés à une diversification des contenus. Il nous apparaît important, dans tout enseignement de l'histoire, de présenter les principales interprétations historiques pour permettre aux élèves d'étudier les grands débats qui ont marqué l'historiographie québécoise. Cette démarche est certainement plus susceptible de développer l'esprit critique des jeunes que celle de fréquenter des sites Internet comme celui d'Historica! Oui, nous voulons que les élèves puis- 
sent évaluer, analyser et critiquer les diverses interprétations construites par la nouvelle historiographie et ainsi leur permettre de se forger une opinion après une connaissance approfondie du dossier. Mais cela ne devrait pas permettre aux partisans d'une histoire rassembleuse de faire table rase des interprétations qui ne sont pas à la dernière mode. Contrairement à ce qu'affirment Dagenais et Laville, nous ne préconisons pas un retour à l'enseignement de "l'idéologie de la survivance» du temps de Groulx. Nous souhaitons seulement que l'élève du secondaire puisse découvrir les études des historiens et historiennes qui ont fait l'histoire au Québec.

Par ailleurs, Dagenais et Laville affirment que nous serions en faveur d'un enseignement de l'histoire où la matière serait "préconstruite autour des "moments fondateurs" ". Pour notre part, sans entrer dans le débat sur le socio-constructivisme, les auteurs ne nous ont pas convaincus qu'il est préférable de laisser l'élève du secondaire construire par lui-même son propre récit historique. Nous n'apprécions pas la caricature qui est faite de l'enseignant «dit traditionnel» qui ne saurait que pratiquer un bourrage de crâne de ses élèves en leur faisant mémoriser des dates, comme si l'approche active de l'élève autour de l'enseignement par projet était née avec la réforme de l'an 2000.

De plus, nous ne sommes pas d'accord avec leur conception simpliste des rapports entre histoire et mémoire. Nos deux auteurs qui s'appuient sur une citation de Pierre Nora pour présenter l'opposition fondamentale entre histoire et mémoire ne semblent pas au courant que de récentes études (voir entre autres l'ouvrage publié sous la direction de M. Meunier et J. Y. Thériault intitulé Les impasses de la mémoire, Fides, 2007) montrent que cette opposition canonique entre histoire et mémoire n'est pas pertinente, parce que le récit mémoriel doit être soumis au regard critique de l'historien dont le rôle est justement de corriger la mémoire. Il doit donc séparer la vérité de la fidélité et c'est en classe que l'enseignant peut corriger les préjugés liés à la mémoire nationale spontanée des jeunes.

Un enseignement de l'histoire pour mieux comprendre le Québec d'aujourd'hui

Vouloir continuer à expliquer aux jeunes Québécois des dates charnières comme 1760, 1840 et 1867 n'est pas synonyme de fermeture et de repli sur soi, au contraire nous croyons qu'une meilleure connaissance de ces événements chez les élèves de toutes origines leur permettra de s'ouvrir aux autres, tout en ayant une meilleure compréhension de la réalité québécoise. Prenons comme exemple la loi 101; si les jeunes 
Québécois issus de l'immigration ignorent le parcours historique de la société québécoise, accepteront-ils aussi facilement de parler français à l'école? Si nous ne leur enseignons plus qu'il y a 400 ans, le territoire du Québec fut colonisé par la France et que nos ancêtres ont été conquis en 1760 par l'Angleterre, ils auront de la difficulté à comprendre pourquoi nous les obligeons à aller à l'école française en 2007. L'ouverture à la diversité ne signifie pas qu'il faille oublier notre histoire qui est le creuset de notre identité. Tout comme la Révolution tranquille, la Conquête de 1760 et la Fédération de 1867 font partie intégrante de l'histoire québécoise et canadienne. Décider de ne plus en parler c'est en quelque sorte renier notre passé. D’ailleurs aucun Québécois issu des communautés culturelles ne nous a demandé de le faire. Tout en étant ouverts à la diversité, il faudrait peut-être que les historiens cessent d'avoir honte d'enseigner l'histoire des Canadiens devenus Canadiens français, nom que portait la majorité québécoise jusqu’en 1960.

\section{UN INTÉRÊT RENOUVELÉ POUR L'ENSEIGNEMENT DE L'HISTOIRE AU SECONDAIRE}

Avec Laville et Dagenais, nous nous réjouissons que cette polémique ait réveillé l'intérêt de plusieurs historiens pour l'enseignement de l'histoire au secondaire, sujet qui n'avait pas mobilisé leur attention depuis bien des années. Ce désintéressement s'explique par le fait que la rédaction et la consultation des programmes d'enseignement de l'histoire sont confiées de plus en plus aux spécialistes des Facultés d'éducation, qui sont éloignés des historiens universitaires. Ce débat aura de façon bénéfique permis aux historiens de métier de s'intéresser à nouveau aux finalités de l'enseignement de l'histoire au secondaire. En outre, même si les historiens universitaires sont moins présents dans la formation des maîtres en histoire, ce que nous déplorons par ailleurs, cela ne signifie pas pour autant qu'ils ont perdu toute capacité d'analyse critique face aux nouveaux programmes.

En conséquence, contrairement à Dagenais et Laville qui prétendent que ceux qui ont dénoncé ce projet de programme étaient inaptes à le faire, car n'ayant qu' « une connaissance superficielle du nouveau programme ", nous croyons au contraire que les historiens avaient tout à fait l'expertise nécessaire pour effectuer une critique en règle de ce programme. Comme si en dehors de la corporation des didacticiens ou autres spécialistes de l'éducation, les historiens ne pouvaient pas porter un jugement éclairé sur le contenu des programmes, ce sur quoi nous avons fait porter notre critique. Nous ne croyons pas qu'il est nécessaire d'être 
didacticien ou conseiller pédagogique pour constater les nombreuses lacunes de ce projet de programme. Alors que Dagenais et Laville s'indignent que de simples enseignants, qui ne sont pas «historiens de métier», aient protesté contre ce projet de programme, loin d'y voir un dérapage nous y voyons une saine réaction citoyenne qui a permis un heureux naufrage.

N'est-il pas étonnant de constater que la majorité des historiens n'ont pas pris la défense du programme? Dans la deuxième phase de consultation, en plein cœur de la polémique, plusieurs historiens universitaires ont acheminé des critiques sévères qui n’ont malheureusement pas été rendues publiques. Même le Conseil d'administration de l'Institut d'histoire de l'Amérique française a formulé de pertinentes critiques que nos deux auteurs n'ont évidemment pas relevées. Finalement, au cours de cette polémique, on a pu découvrir que la presque totalité de ceux et celles qui ont appuyé le projet avaient été associés de près ou de loin à la mise en place de ce programme.

En terminant, nous invitons l'ensemble des historiens à dénoncer la disparition prochaine de l'actuel cours d'histoire facultatif de $V^{\mathfrak{E}}$ secondaire intitulé Connaissance du monde contemporain que le ministère de l'Éducation, du Loisir et du Sport s'apprête à remplacer par un cours obligatoire centré sur les enjeux de l'actualité qui, jusqu'à maintenant, n’a aucune trame historique. Pouvons-nous espérer que les historiens réagiront avant que ce nouveau programme soit adopté et remis aux éditeurs à l'automne 2008 , pour entrer en vigueur en septembre 2009?

(le 4 septembre 2007) 